\title{
Effect of Polysaccharide Species on Storage Stability of Alginate Capsules Containing $\alpha$-Tocopherol
}

\author{
Yusuke Sato, Yoshinari Taguchi, Masato Tanaka* \\ Graduate School of Science and Technology, Niigata University, Niigata-shi, Japan \\ Email: "tanaka@eng.niigata-u.ac.jp
}

Received 4 June 2015; accepted 11 August 2015; published 14 August 2015

Copyright (C) 2015 by authors and Scientific Research Publishing Inc.

This work is licensed under the Creative Commons Attribution International License (CC BY). http://creativecommons.org/licenses/by/4.0/

(c) () Open Access

\begin{abstract}
Alginate capsules containing $\alpha$-tocopherol were prepared adding polysaccharide species such as $\kappa$-carrageenan, gellan gum, pectin, curdlan and ghatti gum. The effects of polysaccharide species and the diameters of $\alpha$-tocopherol on storage stability of the capsules were investigated and characterized in detail. As the contact angle of $\alpha$-tocopherol to the capsule shell strongly affected storage stability, the contact angle was measured on the model sheet made of calcium alginate with polysaccharide species. The leakage ratio decreased with the contact angle and increased with the diameter of $\alpha$-tocopherol. The capsules made of calcium alginate with gellan gum showed the largest contact angle and the highest storage stability. The capsules made of calcium alginate with curdlan, ghatti gum and pectin were harder than other capsules and showed higher storage stability. All the capsules showed the almost similar swelling ratio in the acidic solution with $\mathrm{pH} \mathrm{4}$, but showed the different swelling ratios according to the polysaccharide species in the alkali solution with pH 9. The tablets were prepared with the wet and the dried capsules. The tablets prepared with the dried capsules showed higher storage stability.
\end{abstract}

\section{Keywords}

Calcium Alginate Capsules, $\alpha$-Tocopherol, Gelling in Liquid Method, Polysaccharides, Storage Stability, pH-Responsible Capsules

\section{Introduction}

As the microcapsules have many functions, various kinds of microcapsules have been prepared and applied to

${ }^{*}$ Corresponding author.

How to cite this paper: Sato, Y., Taguchi, Y. and Tanaka, M. (2015) Effect of Polysaccharide Species on Storage Stability of Alginate Capsules Containing $\alpha$-Tocopherol. Journal of Encapsulation and Adsorption Sciences, 5, 111-120. 
the various fields such as cosmetics, food materials, medicine, adhesives, information recording materials, textiles, and agricultural materials [1]-[4].

The main purposes of microencapsulation are to protect the core material from environment, to release the core material according to occasion demands and to handle the gaseous and the liquid core materials as the fine solid particles.

Also, more functions of the microcapsules may be expected by selecting the optimum shell material for the core to microencapsulate.

$\alpha$-tocopherol (VE) is a representative oil soluble antioxidant and a food additive to provide antioxidant role and has a few physiological active effects as a functional material to prevent cardiovascular disease and cancer [5] [6].

However, application of VE is limited, because VE is labile to oxygen and heat. Accordingly, it may be expected that microencapsulating VE can extend the applicable fields.

Many kinds of polysaccharide species have been used to microencapsulate various drugs and physiological active agents and applied to the drug and the food materials [7]-[13].

Among polysaccharides, sodium alginate is a typical capsule shell material and extremely many works about the alginate microcapsules have been reported [11] [12] [14].

S. H. Yoo, et al. have prepared the alginate microcapsules containing $\alpha$-tocopherol with the spray drying method followed by the gelling in-liquid method and investigated the preparation conditions affecting the release properties [5].

They have reported about the operation conditions such as the weight ratio of core to shell, the water soluble emulsifier concentration and the concentration of $\mathrm{CaCl}_{2}$ to optimize the microencapsulation yield.

D. W. Lee, et al. have prepared the alginate microcapsules containing drug (Felodipine) coated and blended with polymers to prevent a rapid release and investigated the effect of structure of microcapsules on the drug release properties [15].

S. K. Bajpai and S. Sharma have investigated swelling/degradation behavior of alginate beads crosslinked with $\mathrm{Ca}^{2+}$ and $\mathrm{Ba}^{2+}$ ions and reported that the alginate beads crosslinked with $\mathrm{Ba}^{2+}$ ions exhibited greater water uptake with increase in acid treatment time [16].

Many reports stated above have almost investigated the effects of preparation conditions affecting the release characteristics. However, there are no reports with regard to the effects of polysaccharides as additives on storage stability and application to the tablet.

The purposes of this study are to prepare the alginate capsules containing $\alpha$-tocopherol by adding polysaccharides, to investigate the effects of polysaccharide species and the diameters of $\alpha$-tocopherol on storage stability, to make the tablets with the capsules and to characterize storage stability.

\section{Experimental}

\subsection{Materials}

Materials used to prepare the capsules and the tablets were as follows.

Sodium alginate (AlNa) and calcium chloride were adopted as the main capsule shell material and the gelling agent, respectively. $\alpha$-tocopherol (VE) was used as the core material. Polysaccharides such as curdlan, $\kappa$-carrageenan, pectin, ghatti gum and gellan gum were used as the additives to improve the mechanical strength of capsule and to control storage stability of core and $\mathrm{pH}$ responsibility. Lactose, calcium stearate and crystalline cellulose were used as the binders to make the tablets. Distilled water was used as the solvent for the materials stated above.

The chemicals stated above were from Wako Pure Chemicals Co., Ltd., Tokyo, Japan.

\subsection{Preparation of Capsules}

Figure 1 shows the flow chart for preparing the capsules together with the schematic diagram of experimental apparatus. The aqueous solution dissolving polysaccharide and AlNa of given weight was stirred with the rotor stator homogenizer by adding VE of core material to form the $(\mathrm{O} / \mathrm{W})$ emulsion. Then, the $(\mathrm{O} / \mathrm{W})$ emulsion was dropped down into the calcium chloride aqueous solution through the nozzle with the diameter of $1.2 \mathrm{~mm}$ by use of syringe pump with the feeding velocity of $60 \mathrm{ml} / \mathrm{h}$ to prepare the capsules (hereafter called as the wet capsules). In this fundamental preparation process, the polysaccharide species were changed. 
A part of the wet capsules was dried in the dryer of $44^{\circ} \mathrm{C}$ for $12 \mathrm{~h}$ to make the dried capsules (hereafter called as the dried capsules). Thus, two kinds of capsules, namely the wet and the dried capsules were prepared and characterized.

The experimental conditions are shown in Table 1.

\subsection{Preparation of Tablets}

Two kinds of the tablets were prepared with the wet and the dried capsules together with binders by changing the pressure with the tableting machine (Ichihashi Seiki Co., Ltd, HANDTAB-100). The sizes of tablet thus prepared were $8 \mathrm{~mm}$ in diameter and $4 \mathrm{~mm}$ in height. The effects of the pressure loaded and polysaccharide species on storage stability were investigated.

\subsection{Characterization}

1) Diameters of VE droplets and capsules

The diameter distributions and mean diameters of VE droplets in the $(\mathrm{O} / \mathrm{W})$ emulsion were measured by the Particle Size Analyzer (SALD-3000: Shimazu Seisakusho, Co., Ltd., Kyoto, Japan). The diameters of capsules were directly measured from the photographs taken by the optical microscope with digital camera (BX51:

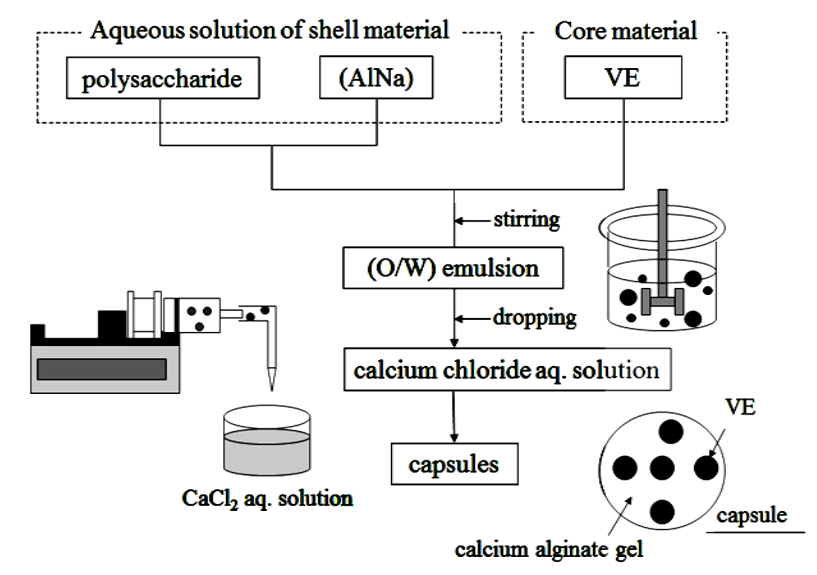

Figure 1. Flow chart for preparing capsules and schematic experimental apparatus.

Table 1. Experimental conditions.

Preparation of shell solution

Distilled water

Sodium alginate (AlNa)

0.6 g (2 wt\%)

$\underline{\text { Polysaccharides }}$

Curdlan, $\kappa$-carrageenan, pectin, ghatti gum, gellan gum

0.3 g (1 wt $\%)$

Preparation of $(\mathrm{O} / \mathrm{W})$ emultion

$\alpha$-tocopherol (VE)

$2.0 \mathrm{~g}$

Revolution speed of homogenizer

$5000 \mathrm{rpm}$

Stirring time

$10 \mathrm{~min}$

Gelling agent

Calcium chloride

$3 \mathrm{wt} \%$

Dropping conditions

Feeding velocity

$60 \mathrm{ml} / \mathrm{h}$

Temperature

$60^{\circ} \mathrm{C}$ 
OLYMPUS Co., Ltd., Kyoto, Japan).

2) Observation of capsules and tablets

The capsules and the tablets were observed by the stereo microscope with the digital camera (SZ1145TR: OLYMPUS Co., Ltd., Tokyo, Japan).

3) Mechanical strength of capsules

Mechanical strength of capsules was measured by the Hardness Meter for the fine particles (DVH-211: Shimazu Seisakusho, Co., Ltd., Kyoto, Japan). The effect of polysaccharide species on mechanical strength was investigated.

4) Contact angle of VE to capsule shell

As storage stability of VE droplets in the capsule is considered to be strongly dependent on wettability of VE to the capsule shell, the contact angle of VE to the capsule shell was measured as follows.

The aqueous solution dissolving AlNa and each polysaccharide was poured on the slide glass $(3 \mathrm{~cm} \times 7 \mathrm{~cm})$ and then, this slide glass was soaked in the calcium chloride aqueous solution for $2 \mathrm{~h}$ at $60^{\circ} \mathrm{C}$ and dried at $44^{\circ} \mathrm{C}$ for $12 \mathrm{~h}$ in order to prepare the model sheet of capsule shell.

Figure 2 shows the processes for preparing the model sheet and measuring the contact angle. VE was dropped on the model sheet through the microsyringe to form a VE droplet. The contact angle of VE to the model sheet was estimated according to the measuring method as shown in Figure 2. Namely, the photograph of the VE droplet on the model sheet was taken by digital camera after elapsing 5 min to make the dynamic equilibrium establish and the contact angle $(\theta)$ was calculated according to the equation as shown in Figure 2.

5) Measurement of VE leaked from capsules

The VE weight leaked from the capsules was measured as follows. After wrapping the wet or the dried capsules of a given weight with the oil absorbable paper, the weight of $200 \mathrm{~g}$ was loaded on this wrap for 10min. Then, the weight of oil absorbable paper was measured and the weight of leaked VE was calculated from difference in the weight of oil absorbable paper.

The leakage ratio (LR) was obtained from the following equation.

$$
\mathrm{LR}=\frac{\text { weight of leaked VE }}{\text { weight of encapsulated VE }} \times 100
$$

6) Swelling ratio of dried capsules

In order to investigate the swelling of dried capsules in the aqueous solution and $\mathrm{pH}$ responsibility, the selling ratios of dried capsules were measured as follows.

The photographs of dried capsules soaked in the aqueous solution with the different $\mathrm{pH}$ value were taken by the optical microscope at the constant time intervals. The transient mean diameters of capsules were directly measured from these photographs. Thus, the swelling ratio (SR) of capsules was estimated by the following equation.

$$
\mathrm{SR}=\frac{d_{p t}-d_{p o}}{d_{p o}} \times 100
$$

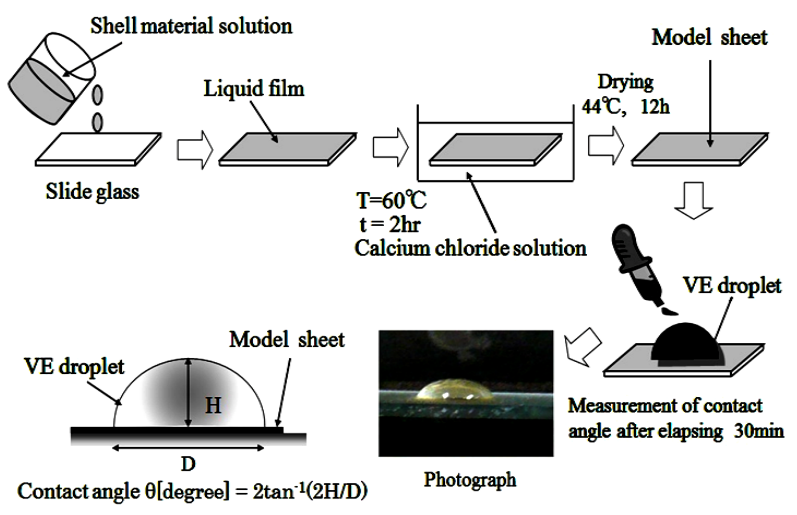

Figure 2. Processes for preparing model sheet and measuring contact angle. 
where $d_{p o}$ and $d_{p t}$ are the diameters of dried capsules at the elapsing time of $t=o$ and $t=t$, respectively.

7) Reduction ratio $(\lambda)$

In order to investigate how the leakage is affected by the process changing from the wet capsules to the dried capsules, the reduction ratios were measured as follows.

The wet capsules were reduced by drying to be the dried capsules. VE should be leaked during the reduction process. So, the reduction ratio $(\lambda)$ for the capsules was estimated by the following equation.

$$
\lambda=\frac{d_{p w}^{3}-d_{p d}^{3}}{d_{p w}^{3}} \times 100
$$

where $d_{p w}$ and $d_{p d}$ are the mean diameters of wet capsules and dried capsules, respectively.

\section{Results and Discussion}

\subsection{Observation of Capsules}

Figure 3 shows the optical microscopic photographs of the wet and the dried capsules prepared by adding each polysaccharide. The sound spherical wet capsules could be prepared under each condition. Furthermore, it is found that the wet capsules have the white smooth surface and the dried capsules have the slightly rough surface due to reduction based on drying.

Also, all the dried capsules become yellow attributing to the reduction of volume of capsules and the VE droplets. Furthermore, the coloring degree is different depending on the capsule shell.

From this result, it may be thought that affinity of VE to the capsule shell, in other words, storage stability is strongly dependent on the physico-chemical properties of capsule shell.

\subsection{Contact Angle of VE to Capsule Shell}

As storage stability of capsules is considered to be strongly depending on affinity of VE to the capsule shell, the contact angle of VE to the capsule shell was measured as an index of affinity. The results are shown in Table 2.

The contact angles were $28^{\circ}$ ( $\kappa$-carrageenan), $43^{\circ}$ (ghatti gum), $58^{\circ}$ (curdlan), $62^{\circ}$ (pectin), $75^{\circ}$ (gellan gum) in turn. The capsule shell made of AlNa with gellan gum shows the largest contact angle. It may be expected that the larger the contact angle, the higher the storage stability.

Accordingly, it is necessary to investigate how the degree of storage stability is depending on the capsule shell.

\subsection{Dependence of Leakage Ratio of Wet Capsules on Polysaccharide Species}

Figure 4 shows the dependence of leakage ratio (LR) of the wet capsules on the polysaccharide species, where the leaked weight of VE was measured by changing the load required to constrict the diameter of $d_{p}$ to one half $\left(d_{p} / 2\right)$ of the diameter as shown in Figure 4. From the figure, it is found that mechanical strength and the leakage ratios were different according to the polysaccharide species. The leakage ratios of all the wet capsules were not measured for ca. $1 \mathrm{~h}$ at loadless $(\mathrm{W}=\mathrm{O})$ as shown in Figure 4. This result may be due to the fact that water contained in the capsule shell should prevent VE from leaking. The capsules made of AlNa with ghatti

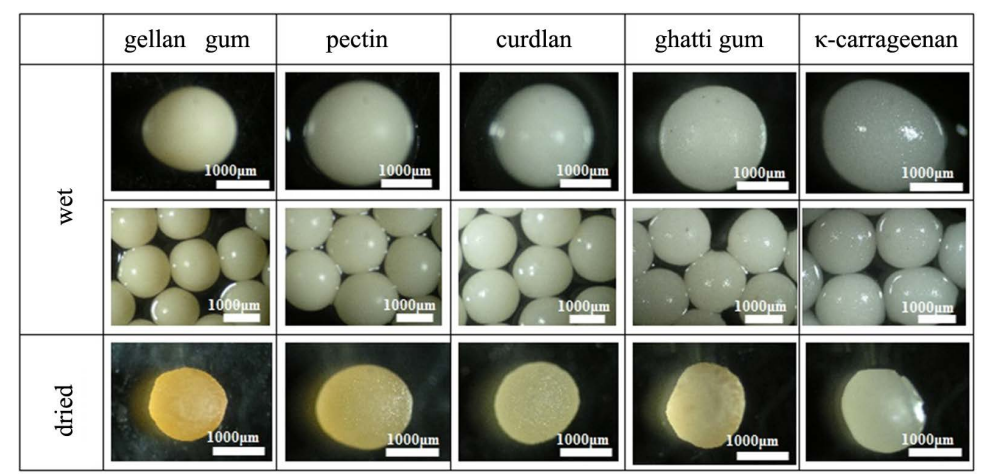

Figure 3. Observation of wet and dried capsules. 


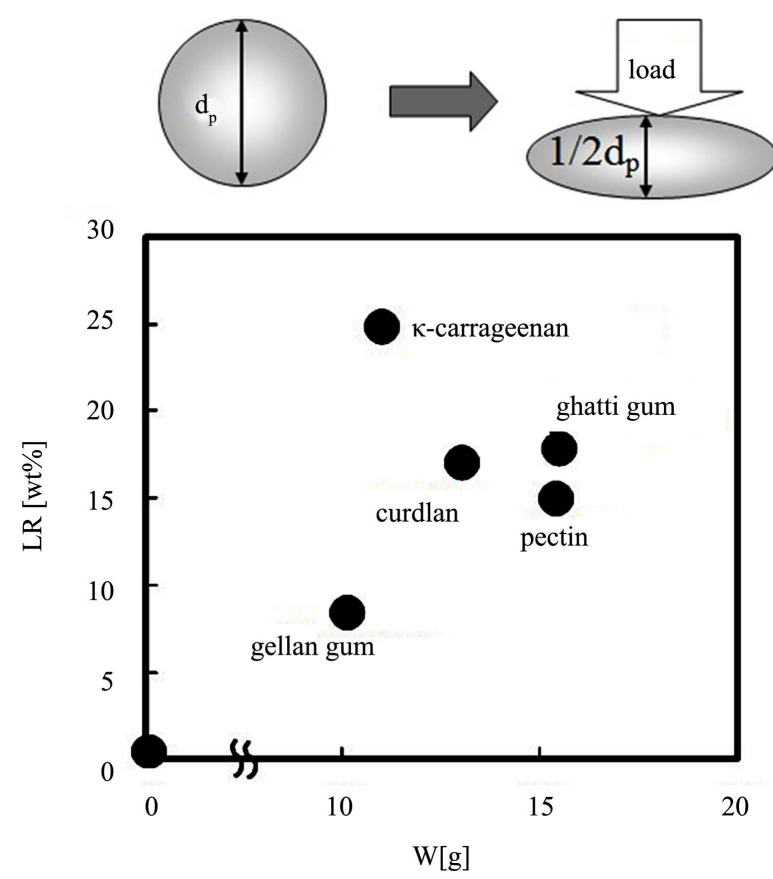

Figure 4. Dependence of leakage ratios on polysaccharide spacies.

Table 2. Experimental conditions.

\begin{tabular}{cccccc} 
& & \multicolumn{3}{c}{ Polysaccharide } \\
& $\kappa$-carrageenan & Ghatti gum & Curdlan & Pectin & Gellan gum \\
\hline$\theta$ [degree] & 28 & 43 & 58 & 62 & 75 \\
\hline
\end{tabular}

gum and pectin were stronger than those with other polysaccharides, because the load required to constrict toone half of the diameter were larger. Meanwhile, the capsules made of AlNawith $\kappa$-carrageenan show the highest leakage ratio (25\%) at the load of $11.0 \mathrm{~g}$. This result may be due to the lowest contact angle, in other words, the highest affinity of VE to the capsules shell.

\subsection{Dependence of Leakage Ratio of Dried Capsules on Contact Angle and VE Droplet Diameter}

The dependence of leakage ratio of dried capsules on the contact angle is shown in Figure 5. The leakage ratio decreased with the contact angle and become the highest for the dried capsules made of AlNa with $\kappa$-carrageenan showing $\theta=28^{\circ}$ and the lowest for the dried capsules made of AlNa with gellan gum showing $\theta=75^{\circ}$, respectively. From these results, it is found that storage stability of the dried capsules could be controlled by the polysaccharides species, too.

Also, storage stability should be affected by stability of VE droplets in the capsule. In general, it is well known that stability of VE droplets in the capsules may be strongly affected by the VE droplet diameter [17] [18]. Namely, the smaller the VE droplets, the higher the dispersion stability. So, as the diameter of VE droplet in the $(\mathrm{O} / \mathrm{W})$ emulsion may be considered to largely affect the leakage ratio, the dependence of leakage ratio for the dried capsules on the VE droplet diameter $\left(d_{p}\right)$ is shown in Figure 6. The diameter of VE droplets in the shell solution made of AlNa with ghatti gum is larger than in the other shell solution. The diameters of dispersed droplets are different according to the physical properties of liquids concerned mainly such as interfacial tension and viscosity. For examples, $d_{p} \sim \gamma^{0.35 \sim 0.75} \mu_{\mathrm{c}}^{-0.15} \mu_{\mathrm{d}}^{0.15}$, where $\gamma, \mu_{\mathrm{c}}, \mu_{\mathrm{d}}$ are interfacial tension, viscosity of continuous phase and dispersed phase, respectively [19]. It is found that the leakage ratio increased with the VE droplet diameters except the capsules made of AlNa with $\kappa$-carrageenan. As it is well known that the oil droplet 

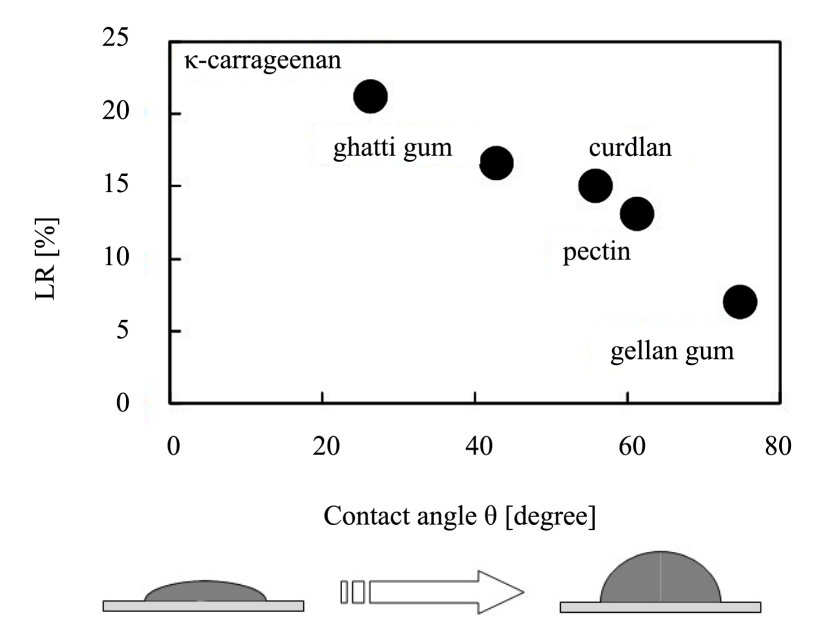

Figure 5. Dependence of leakage ratio of dried capsule on contact angle.
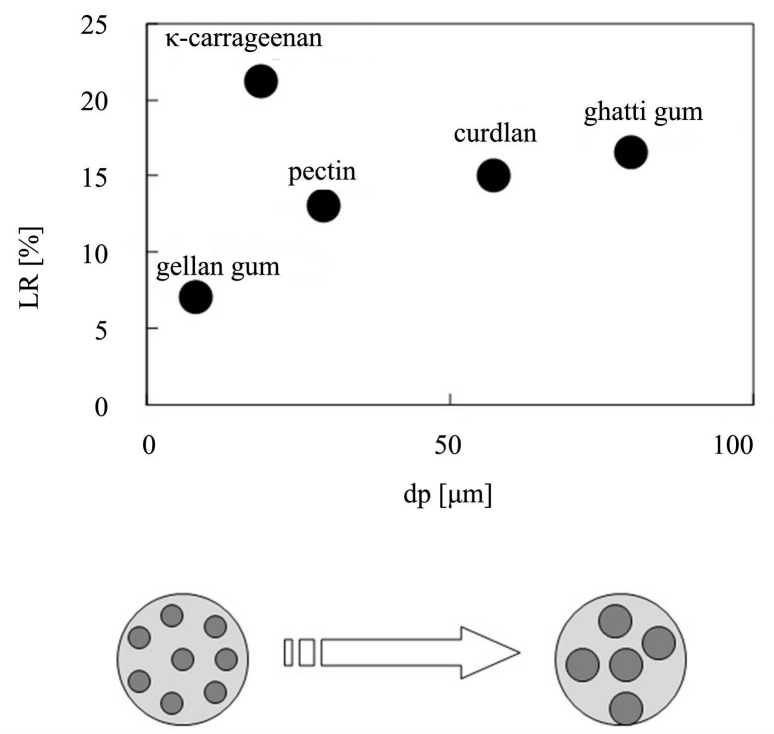

Figure 6. Dependence of leakage ratio of VE droplet diameter.

diameters increase due to coalescence between the droplets in the unstable $(\mathrm{O} / \mathrm{W})$ emulsion, the leakage ratio may increase with the diameters of unstable VE droplets. The leakage ratio for the dried capsules made of AlNa with $\kappa$-carrageenan may become larger due to the lower contact angle as shown in Figure 5. Namely, as affinity between the capsule shell and VE is larger, VE may permeate comparatively easily through the capsule shell.

\subsection{Selling of Capsules}

Figure 7 shows the transient swelling ratios (SR) of dried capsules soaked in the solution with $\mathrm{pH} 4$ (a) and $\mathrm{pH}$ 9 (b). In the solution with $\mathrm{pH} \mathrm{4}$, all the capsules showed the almost similar transient swelling ratio, namely all the capsules similarly swelled from $0 \%$ to $100 \%$. However, in the solution with $\mathrm{pH} 9$, the transient swelling ratios were different according to the polysaccharide species. Namely the capsules made of AlNa with curdlan and gellan gum swelled from $0 \%$ to $5 \%$ at the most. While, the capsules made of AlNa with $\kappa$-carrageenan, pectin and ghatti gum swelled from $0 \%$ to $15 \%, 17 \%$ and $22 \%$, respectively.

At all events, the swelling ratios for the dried capsules in the solution with $\mathrm{pH} 9$ are considerably smaller than those in the solution with $\mathrm{pH} 4$.

These results may mean that each capsule made of AlNa with polysaccharide species shows different $\mathrm{pH}-$ 
responsiblity. It is very important to know the informations about the leakage ratio on the basis of changing from the wet capsules to the dried capsules. The diameters of wet capsules may decrease with the drying time and become the diameters of dried capsules. So, the reduction ratio $(\lambda)$ defined by Equation (3) may be different according to the capsule shell.

Figure 8 shows the dependence of leakage ratio on the reduction ratio for each capsule. The leakage ratio increased with the reduction ratio. The capsule made of AlNa with $\kappa$-carrageenan showing the larger reduction ratio shows the higher leakage ratio. From this result, it is found that storage stability could be improved by polysaccharide species, and the capsules made of AlNa with gellan gum showed the highest storage stability.

\subsection{Preparation of Tablets and Observation}

As an example for applying the capsules, it is usually tried to make the tablets with the capsules and binders. In order to obtain the informations about storage stability of VE in the tablet, the tablets were made of the capsules and binders, and leakage feature of VE was observed by changing the load on the tablet.

Figure 9 shows the results observing the tablets made of capsules and binders, where Figure 9(a) and Figure 9(b) are the tablets with the wet capsules and Figure 9(c) and Figure 9(d) the tablets with the dried capsules, respectively. Also, Figure 9(a) and Figure 9(c) are the side views of tablets and Figure 9(b) and Figure 9(d) the top views of tablets. Furthermore, the three tablets in each photograph were loaded by $0.5 \mathrm{t}, 1.0 \mathrm{t}, 1.5 \mathrm{t}$ in turn. As the color on the side views of tablets prepared with the wet capsules largely changed, it is found that large VE was leaked. On the other hand, the tablets prepared with the dried capsules showed no leakage and the higher storage stability except the dried capsules made of AlNa with $\kappa$-carrageenan. Accordingly, storage stability for the tablets prepared with the wet capsules is lower and that for the tablets prepared with the dried capsules is higher.

As gellan gum and pectin are gelled with calcium ion, the capsules shell made of AlNa with these polysaccharides may be prepared by cogelling with sodium alginate and these polysaccharides.

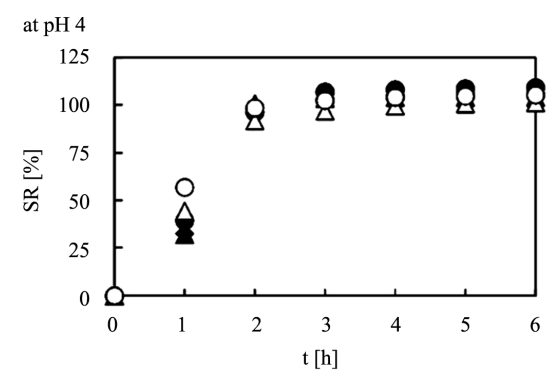

(a)

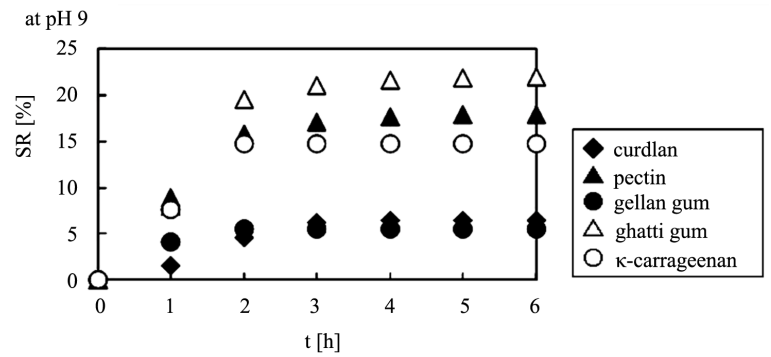

(b)

Figure 7. Transient swelling ratio of dried capsules.

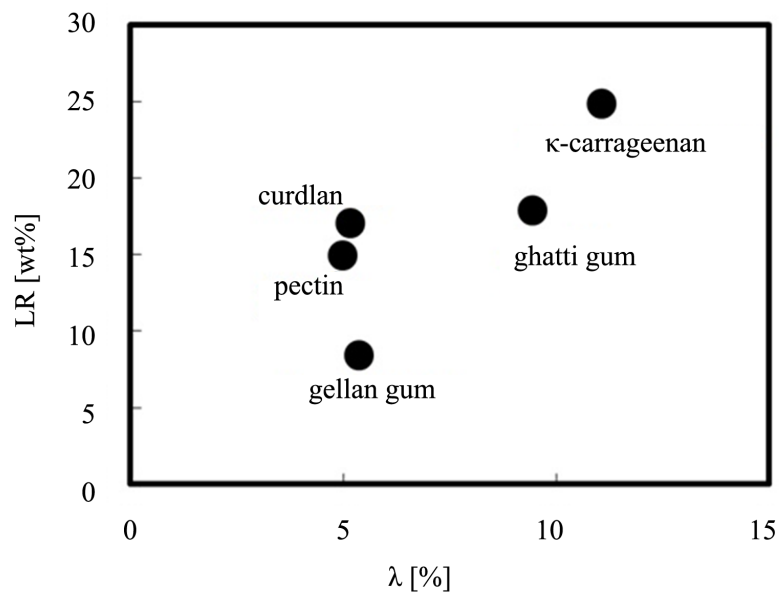

Figure 8. Dependence of leakage ratio on reduction ratio. 


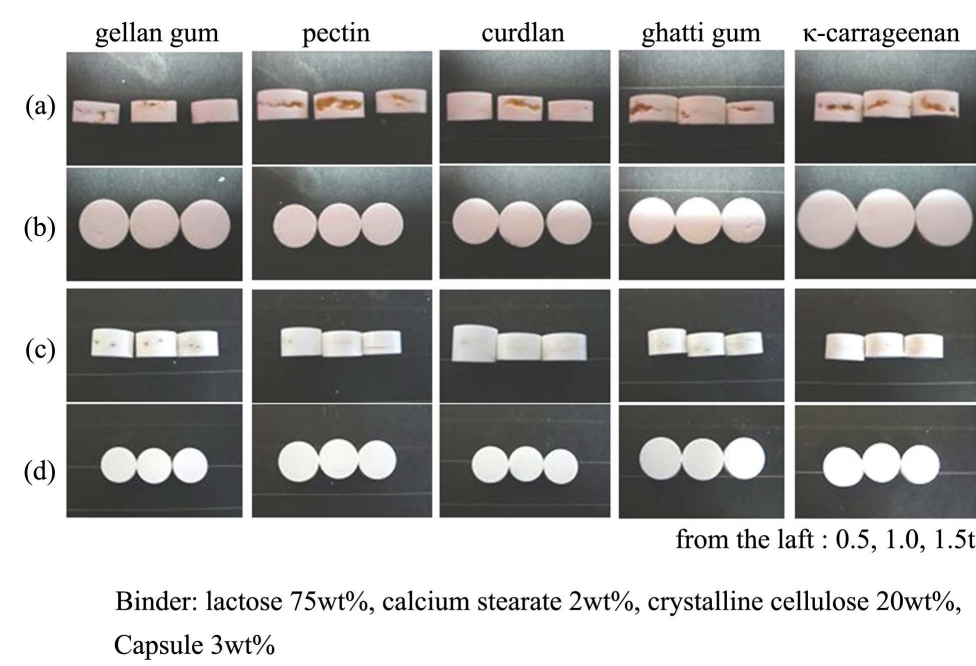

Figure 9. Observation of tablets made of capsules and binders.

Also, as curdlan gels by heating over $80^{\circ} \mathrm{C}$ to form strong gel, the capsules shell made of AlNa with curdlan may be prepared by gelling with calcium ion and heating gelatinization.

As gattigum and $\kappa$-carrageenan gel at room temperature, the capsule shell made of AlNa with these polysaccharides may be prepared by gelling with calcium ion and self-gelatinization.

Storage stability of the capsules made of AlNa with polysaccharides may be affected by the structure, mechanical strength and physical properties of capsule shell.

Accordingly it is necessary to investigate especially how the structure and physical properties of capsule shell affect storage stability in detail.

\section{Conclusions}

The capsules made of alginate with polysaccharides containing $\alpha$-tocopherol were prepared and the tablets were prepared with the capsules and binders. The effect of polysaccharide species on storage stability was investigated and the following fundamental results could be obtained.

1) The leakage ratio decreased with the contact angle of $\alpha$-tocopherol to the capsule shell.

2) The capsules made of calcium alginate with gellan gum showed the largest contact angle to $\alpha$-tocopherol and the lowest leakage ratio.

3) The capsules made of calcium alginate with gellan gum were the softest capsule.

4) All the capsules similarly swelled from 0 to $100 \%$ in the aqueous solution with $\mathrm{pH} 4$.

5) The capsules showed different $\mathrm{pH}$ responsibility in the aqueous solution with $\mathrm{pH} 9$.

6) All the tablets made of wet capsules and binders showed the larger leakage of VE and lower storage stability.

7) The tablets made of dried capsules and binders showed higher storage stability except the dried capsules made of AlNa with $\kappa$-carrageenan.

\section{References}

[1] Kondo, T. and Tanaka, M. (1975) Microcapsules (Preparation, Properties, Application). Sankyo Shuppan, Tokyo.

[2] Kondo, T. (1967) Saishin Maikurokapseruka Gijutsu (Microencapsulation Technique). TES, Tokyo.

[3] Tanaka, M. (2008) Key Point of Preparation of Nano/Microcapsules. Techno System Publishing Co. Ltd., Tokyo.

[4] Koishi, M., Eto, K. and Higure, H. (2005) (Preparation + Utilization) Microcapsules. Kogyo Chosakai, Tokyo.

[5] Yoo, S.H., Song, Y.B., Chang, P.S. and Lee, H.G. (2006) Microencapsulation of $\alpha$-Tocopherol Using Sodium Alginate and Its Controlled Release Properties. International Journal of Biological Macromolecules, 38, 25-30. http://dx.doi.org/10.1016/j.ijbiomac.2005.12.013

[6] Song, Y.B., Lee, J.S. and Lee, H.G. (2007) $\alpha$-Tocopherol-Leaded Ca-Pectinate Microcapsules. Colloids and Surfaces B: Biointerfaces, 73, 394-398. http://dx.doi.org/10.1016/j.colsurfb.2009.06.014 
[7] Dou, H., Tang, M. and Sun, K. (2005) A Facile One-Pot Synthesis to Dextran-Based Nanoparticles with Carboxy Functional Groups. Macromolecular Chemistry and Physics, 206, 2177-2181. http://dx.doi.org/10.1002/macp.200500326

[8] Ma, J., Xu, Y., Fan, B. and Liang, B. (2007) Preparation and Characterization of Sodium Carboxymethylcellulose/ Poly(N-Isopropylacrylamide)/Clay Semi-IPN Nanocomposite Hydrogels. European Polymer Journal, 43, 2221-2228. http://dx.doi.org/10.1016/j.eurpolymj.2007.02.026

[9] Dou, H., Tang, M., Yang, W. and Sun, K. (2007) One-Pot Synthesis, Characterization, and Drug Loading of Polysaccharide-Based Nanoparticles with Carboxy Functional Groups. Colloid Polymer Science, 285, 1043-1047. http://dx.doi.org/10.1007/s00396-007-1657-1

[10] Dulong, V., Mocanu, G. and Cerf, D.L. (2007) A Novel Amphiphilic pH-Sensitive Hydrogel Based on Pullulan. Colloid Polymer Science, 285, 1085-1091. http://dx.doi.org/10.1007/s00396-007-1655-3

[11] Cha, D.S., Choi, J.H., Chinnan, M.S. and Park, H.J. (2002) Antimicrobial Films Based on Na-Alginate and $\kappa$-Carrageenan. LWT-Food Science and Technologie, 35, 715-719. http://dx.doi.org/10.1006/fstl.2002.0928

[12] Denkbas, E.B., Özdemýr, N., Öztürk, E., Eroglu, M. and Acar, A. (2004) Mitomycin-C-Loaded Alginate Carriers for Bladder Cancer Chemotherapy. Journal of Bioactive and Compatible Polymers: Biomedical Applications, 19, 33-34. http://dx.doi.org/10.1177/0883911504041607

[13] Tari, Ö. and Pekcan, Ö. (2004) A Percolation Approach for Investigating the Sol-Gel Phase Transition of $\kappa$-Carrageenan: A Steady-State Fluorescence Study. Journal of Bioactive and Compatible Polymers: Biomedical Applications, 19, 491-509. http://dx.doi.org/10.1177/0883911504048328

[14] Ramesh Babu, V., Krishna Rao, K.S.V., Sairam, M., Vijaya Kumar Naidu, B., Hosamani Kallappa, M. and Aminabhavi Tejraj, M. (2006) pH Sensitive Interpenetrating Network Microgels of Sodium Alginate-Acrylic Acid for the Controlled Release of Ibuprofen. Journal of Applied Polymer Science, 99, 2671-2678. http://dx.doi.org/10.1002/app.22760

[15] Lee, D.W., Hwang, S.J., Park, J.B. and Park, H.J. (2003) Preparation and Release Characteristics of Polymer-Coated and Blended Alginate Microspheres. Journal of Microencapsulation, 20, 179-192. http://dx.doi.org/10.3109/02652040309178060

[16] Bajpai, S.K. and Sharma, S. (2004) Investigation of Swelling/Degradation Behaviour of Alginate Beads Crosslinked with $\mathrm{Ca}^{2+}$ and $\mathrm{Ba}^{2+}$ Ions. Reactive \& Functional Polymers, 59, 129-140. http://dx.doi.org/10.1016/j.reactfunctpolym.2004.01.002

[17] Morita, R., Saito, N., Taguchi, Y. and Tanaka, M. (2015) Preparation of Microcapsules Containing Phase Change Material and Silicon Carbide Powder with Interfacial Polycondensation Reaction Method. Materials Sciences and Applications, 6, 251-262. http://dx.doi.org/10.4236/msa.2015.63030

[18] Koyama, Y., Saito, N., Fuchigami, K., Taguchi, Y. and Tanaka, M. (2015) Microencapsulation of Sodium Hydrogen Carbonate to Generate Carbon Dioxide with Thermal Responsible Shell Material. Journal of Cosmetics, Dermatological Sciences and Applications, 5, 36-44. http://dx.doi.org/10.4236/jcdsa.2015.51005

[19] Oshima, E. and Tanaka, M. (1982) Coalescence and Break up of Droplets in Suspension Polymerization of Styrene. Kagaku Kogakuronbunshu, 8, 86-90. http://dx.doi.org/10.1252/kakoronbunshu.8.86 